\title{
Retour d'expérience sur les inondations dans l'agglomération de Sfax (Tunisie méridionale) de 1982 à 2009 : de la prévention à la territorialisation du risque
}

Feedback on flooding in the city of Sfax (southern Tunisia) from 1982 to 2009:

from prevention to the territorialization of risk

\section{Abdelkarim Daoud}

\section{OpenEdition}

Journals

Édition électronique

URL : http://journals.openedition.org/rge/4630

DOI : $10.4000 /$ rge.4630

ISSN : 2108-6478

Éditeur

Association des géographes de l'Est

Édition imprimée

Date de publication : 31 août 2013

ISSN : 0035-3213

Référence électronique

Abdelkarim Daoud, «Retour d'expérience sur les inondations dans l'agglomération de Sfax (Tunisie méridionale) de 1982 à 2009 : de la prévention à la territorialisation du risque », Revue Géographique de l'Est [En ligne], vol. 53 / 1-2 | 2013, mis en ligne le 16 septembre 2013, consulté le 08 septembre 2020. URL : http://journals.openedition.org/rge/4630 ; DOI : https://doi.org/10.4000/rge.4630

Ce document a été généré automatiquement le 8 septembre 2020

Tous droits réservés 


\section{Retour d'expérience sur les inondations dans l'agglomération de Sfax (Tunisie méridionale) de 1982 à 2009 : de la prévention à la territorialisation du risque}

Feedback on flooding in the city of Sfax (southern Tunisia) from 1982 to 2009 : from prevention to the territorialization of risk

\section{Abdelkarim Daoud}

\section{Introduction}

Située dans une plaine basse vers laquelle convergent plusieurs cours d'eau, l'agglomération de sfax a connu durant les quatre dernières décennies plusieurs évènements pluvieux qui provoquèrent des crues et stagnations d'eau causant parfois de graves dommages aux personnes et aux biens. A travers une étude rétrospective, deux évènements exceptionnels sont retenus dans la présente étude : les crues de 1982 et celles de 2009. L'objectif est de montrer les limites des aménagements lourds et coûteux de protection contre les inondations, surtout en raison de l'évolution spatiale et temporelle des vulnérabilités. Dans une première partie seront traités les éléments expliquant la vulnérabilité naturelle de l'agglomération de Sfax, et de son aggravation par les modes inadéquats d'occupation des sols expliquant les graves dégâts générés par les inondations de 1982, et la mise en place des infrastructures d'hydraulique urbaine qui s'en suivirent. Le travail traite ensuite des différenciations spatiales actuelle des vulnérabilités, particulièrement entre la zone centrale et péricentrale à forts enjeux socio-économiques d'une part, et la zone périurbaine à habitat souvent anarchique d'autre part, mettant en relief le dysfonctionnement du système urbain en général comme principale cause de vulnérabilité. Le travail présente enfin des éléments 
de réflexion pour une meilleure articulation entre risque et territoire, proposant d'agir sur les vulnérabilités pour réduire les risques d'inondation en milieu urbain.

\section{Méthodologie}

2 Le présent travail présente une approche géographique des risques d'inondation en milieu urbain, et ce à partir de l'étude du cas de l'agglomération de Sfax, en Tunisie méridionale. A l'approche "classique " à travers l'étude des aléas et vulnérabilités ajoutés aux enjeux, pour expliquer les risques d'inondation, notre méthode s'appuie également sur une approche " territoriale ", visant à déterminer l'évolution des vulnérabilités en rapport avec le fonctionnement (ou dysfonctionnement) de l'espace urbain dans ses différentes composantes. Cette dernière approche nous semble justifier la nécessité d'agir aujourd'hui vers une réduction des vulnérabilités urbaines à travers les différents outils de gestion urbaine, puisque, pour notre cas d'étude, les mesures structurelles (aménagements d'hydraulique urbaine) montrent des limites face à des vulnérabilités spatialement différenciées Outre les observations de terrain, continues depuis quatre décennies environ, le travail ici proposé s'appuie sur les données fournies par l'Institut National de la Météorologie pour les inondations de 1982 et sur les mesures effectuées à l'Ecole Nationale d'Ingénieurs de Sfax pour l'épisode de 2009. Une couverture topographique au 1/50.000 fut également digitalisée et géoréférencée pour déterminer, à partir d'une approche hydrogéomorphologique, le bassin à risque d'inondation de l'agglomération de Sfax. Concernant le bâti, des images Landsat TM (2003 et 2004) furent utilisées pour cartographier l'étalement de l'agglomération et la localisation des quartiers périurbains, particulièrement ceux soumis au risque d'inondation. La synthèse relative aux relations entre risques d'inondation et territoire s'appuie sur la bibliographie disponible, ainsi qu'une réflexion sur les modes de fonctionnement du système urbain à Sfax et des choix d'aménagement opérés jusqu'alors.

\section{Résultats}

\section{La vulnérabilité physique liée au site et les modes d'occupation des sols: raisons majeures des dégâts des inondations de 1982.}

\section{L'aléa générateur. une situation de « retour d'Est » et des pluies exceptionnelles}

Dans les zones semi-arides ou arides, aux années de sécheresse peuvent succéder des événements pluviométriques exceptionnels, voire des catastrophes climatiques. Si la moyenne annuelle pour la station de Sfax-El-Maou pour la période allant de 1901 à 1985 est de 215 millimètres (Daoud A. 1992), celle-ci peut descendre en deçà de 100 millimètres : 37.1 en 1946/47; 52.5 en 1936/37, ou atteindre des chiffres records : 604.4 en 1969/70 ; 430 en 1948/49; 414 en 1975/76. Le caractère de variabilité des pluies se retrouve à l'échelle mensuelle, saisonnière et annuelle. A titre d'exemple, le mois d'octobre a reçu $462.6 \mathrm{~mm}$ en 1969 et $0 \mathrm{~mm}$ en 1910 et 1970. A la lumière des inondations historiques connues de mémoire d'homme, et ayant causé le plus de dégâts en milieu urbain, celle d'octobre 1982 peut être considérée comme crue de référence. Provoquées par une circulation de type NE (Daoud A. 1992.) les inondations subies par 
l'agglomération de Sfax en 1982 furent les conséquences des pluies reçues entre le 30 et le 31 octobre. En effet, les quantités enregistrées en 24 heures le 30 octobre 1982 avaient atteint 140.8 millimètres (période de retour égale à 160 ans), les quantités enregistrées entre le 30 octobre à 22 heures et le lendemain à 11 heures 05 heures avaient atteint 177,3 millimètres (période de retour égale à 500 ans) (Sakis N. et Sassi N. 1982.). Une intensité maximum de 45,2 millimètres par heure fut enregistrée le 31 octobre. Mais bien que le total du mois d'octobre 1969 (457,6 millimètres dont 150,2mm en 24 heures le 7 du mois et 126,7 millimètres le 21) soit plus élevé que celui de 1982, les dégâts qui suivirent les inondations en milieu urbain ne furent pas aussi catastrophiques. En effet, si les pluies de septembre 1969 furent exceptionnelles par leur quantité, celles d'octobre 1982 le furent par leurs intensités. Pour ces dernières, les chiffres officiels estimaient les pertes matérielles à 47 millions de Dinars et les pertes humaines à 70 personnes. Presque trente ans après, les laisses de crues sont encore visibles dans les lits d'oueds ou par certaines ruines. De même, des repères sont encore matérialisés sur certains murs de maisons, marquant le niveau atteint par l'eau. L'émotion engendrée par ces dégâts, ajoutée à une forte médiatisation, avait alors amené le pouvoir politique, au plus haut sommet de l'Etat, à doter l'agglomération de Sfax d'une infrastructure de protection contre les inondations. L'ouvrage principal (le canal de ceinture) fut financé par la Banque Mondiale.

\section{La vulnérabilité physique: Sfax est une plaine où convergent plusieurs oueds}

4 Notre périmètre d'étude ou bassin à risque (Fig. 1) occupe un site de plaine littorale à très faible pente. Celle-ci est comprise entre 0.3 et $1 \%$, mais souvent inférieure à $0.5 \%$, particulièrement dans les secteurs les plus proches de la côte (Oueslati A. 1999.). En raison de leur configuration, les plaines littorales sont souvent exposées aux inondations. Le cas de Sfax n'est pas unique en Tunisie, mais l'acuité du problème vient $\mathrm{du}$ fait que cette plaine abrite une agglomération dépassant 500.000 habitants, occupant parfois de façon anarchique les zones les plus exposées aux effets des crues. Ceinturée par des reliefs de collines de faibles altitudes (Bouaziz R. 2011), la plaine de Sfax est l'exutoire d'un réseau hydrographique peu hiérarchisé, à écoulement épisodique, convergeant souvent vers les zones occupées par l'urbanisation.

5 L'oued Sidi-Salih draine toute la partie nord du bassin et est constitué par un collecteur principal, coulant d'Ouest en Est, et recevant les apports de plusieurs autres petits oueds (oued Sidi-Abdelkafi, oued Saguiet-Zriba, oued Al-Kharrouba), provenant des reliefs de collines ceinturant le bassin. Dans sa partie aval, l'oued Sidi-Salih prend l'appellation d'oued Laachèche. Son lit s'élargit très sensiblement en raison de la faiblesse de la pente. Le secteur de Chott-El-Merdassia et les abords du village de Sidi Mansour constituent alors sa zone d'expansion des crues. Les crues de cet oued peuvent inonder tous les terrains bas situés près de son exutoire, et occupés par l'habitat spontané.

6 L'oued Ezzit, dont le bassin versant ne dépasse pas une trentaine de $\mathrm{km}^{2}$, collecte les eaux du secteur d'El-Medass, situé au NW de Sakiet Ezzit, coule ensuite vers le SE en traversant les communes de Sakiet Ezzit et Sakiet Eddaïr pour s'appeler ensuite oued el Kiblaoui, avant de se jeter, lors des épisodes pluviométriques importants, dans la mer au niveau d'Essaloum, sur le littoral de Sidi Mansour. L'oued El-Awabid draine la partie centrale de notre secteur d'étude et reçoit en amont les écoulements en provenance de Aïn-Torkia. Dans sa section moyenne et aux abords de l'agglomération de Sfax, il va 
s'appeler oued Chaâbouni. Endigué sur sa rive gauche, celui-ci va recevoir les apports de l'oued Agareb. L'ensemble est canalisé jusqu'à l'oued El-Maou pour rejoindre la mer.

Fig.. 1 : limites du bassin à risque d'inondation de l'agglomération de Sfax

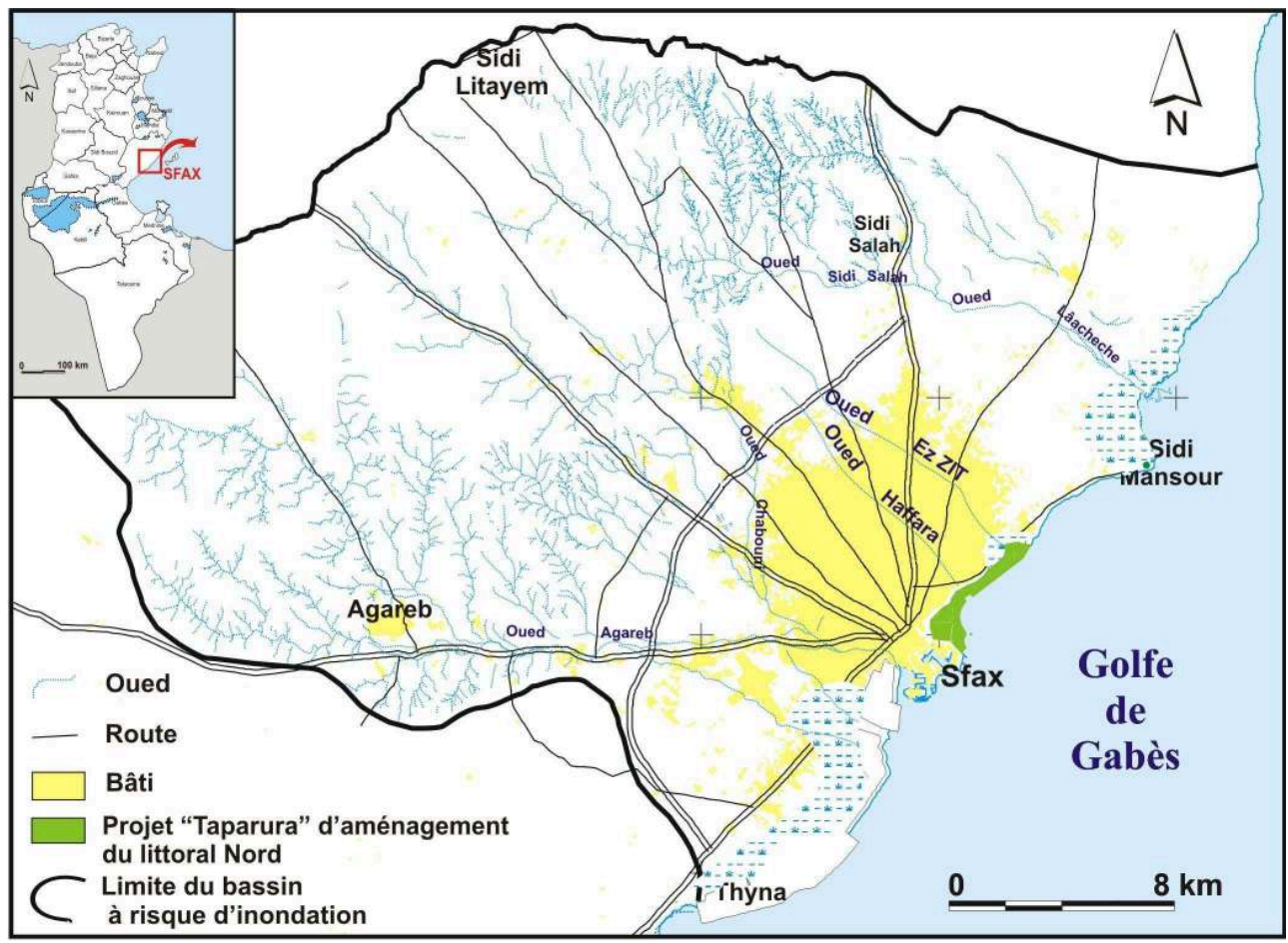

7 L'agglomération de Sfax proprement dite est traversée par de petits oueds dont les lits sont aujourd'hui complètement occultés par l'urbanisation, mais dont les tracés peuvent être retrouvés sur les anciens fonds topographiques au 1/50.000. Le plus important est certainement l'oued El-Haffara, constituant la partie aval de l'oued ZankatEl-Azouza, qui prend naissance en amont de la localité Chihia. Cet ensemble draine les anciens vergers de Sfax, occupés aujourd'hui par un habitat pavillonnaire dense, résultat de leurs morcellements successifs. Par ailleurs, l'oued R'mel, passant par les quartiers populaires à l'ouest de l'agglomération, est aujourd'hui branché dans sa partie aval sur le canal de ceinture. En raison de la taille minuscule de leur bassin versant, certains de ces oueds ne sont pas représentés sur la figure 1.

\section{Des modes d'urbanisation aggravant la vulnérabilité.}

Durant la décennie qui précéda les inondations de 1982, l'agglomération de Sfax avait connu une forte croissance urbaine, particulièrement consommatrice d'espace puisqu'elle privilégie le type d'habitat pavillonnaire isolé (Karray N. 1983; Megdiche T. 1985.). En raison de l'imperméabilisation des espaces urbanisés, les coefficients de ruissellement augmentent, puisqu'un même épisode pluvieux, qui passait autrefois inaperçu et sans dégâts, provoque désormais des ruissellements importants et très violents, en raison de l'accroissement des vitesses de transfert des écoulements, sur des surfaces occupées par les logements, l'infrastructure et parfois même les équipements collectifs. Cette situation entraîna en 1982 des dégâts matériels et humains très importants, suite à l'obstruction des lits d'oueds ou des ruisseaux et à la réduction importante de la capacité d'évacuation des axes d'écoulement naturels (Daoud A. 2005). 
Les dégâts furent d'autant plus graves que les aménagements antérieurs avaient ignoré, parfois délibérément, les circuits naturels de l'eau de ruissellement ou la sensibilité aux inondations de certains îlots urbains et péri-urbains. Pourtant, dans beaucoup de secteurs comme le littoral de Sidi-Mansour, les environs de l'embouchure des oueds ElMaou et Agareb, les berges de l'oued Ezzit, le risque était présent avant l'urbanisation des talwegs et zones basses. C'est pour cette raison que, paradoxalement, les dégâts les plus spectaculaires furent provoqués par les plus petits oueds « urbains » : L'oued Ezzit avait causé des pertes en vies humaines et des dégâts matériels très importants, lors des inondations de 1982. L'obstruction de son lit par les constructions, n'avait pas facilité l'évacuation des débits impressionnants qu'il avait coulés. Lors de ces inondations, embâcles et ruptures d'embâcles se succédèrent en quelques heures, aggravant encore les dégâts sur les berges urbanisées. En fait, l'oued n'avait fait que reprendre ses droits en démolissant tout sur son passage. Sur les 8500 logements endommagés et les 901 détruits dans la région de Sfax suite à ces inondations (Bourgou M. et Oueslati A. 1984), la majorité se trouvait sur le passage de cet oued. De même, le petit oued d'El-Haffara, jusque-là inactif car possédant un micro bassin versant au milieu des vergers à habitat pavillonnaire de l'agglomération, causa des dégâts importants, témoignant des très forts débits qu'il a fait transiter vers le littoral de Sidi Mansour.

9 Par ailleurs et en zone périurbaine, ce sont des oueds relativement importants et non maîtrisés qui ont causé les dégâts les plus importants lors des inondations de 1982. L'oued Laâchèche, constituant la partie aval de l'oued Sidi-Salah, a inondé toute la zone littorale à la périphérie du village côtier de Sidi-Mansour, déjà sous-équipé et à habitat anarchique proliférant dans les zones basses inondables. Les oueds Awabid, Châabouni, El-Maou, et Agareb avaient inondé les secteurs périurbains à l'ouest de l'agglomération.

Il est enfin important de signaler qu'en dehors des terrains occupés par l'urbanisation, les formations superficielles dominantes du bassin à risque d'inondation de Sfax sont des formations sableuses ou sablo-limoneuses meubles et filtrantes probablement d'âge Holocène. Toutefois, les changements introduits par l'occupation humaine, particulièrement les défrichements de la végétation naturelle (jujubier surtout), et la mise en place de plantations d'oliviers, ont entraîné des modifications dans le mode de fonctionnement de ces formations superficielles vis à vis des écoulements. Cela se manifeste particulièrement par l'augmentation des coefficients de ruissellement et les ravinements. De plus, chaque épisode pluvieux important introduit des changements morphologiques sur les faciès. Ainsi, les inondations de 1982 avaient particulièrement entraîné des dynamiques érosives marquées surtout par l'approfondissement et l'élargissement des lits d'oueds, surtout dans les sections où ceux-ci sont traversés par des routes sans ponts, créant ainsi une rupture artificielle de pente (idem), comme c'est le cas de l'oued Chaâbouni à son intersection avec la route de Menzel Chaker. Il est clair que les conditions d'écoulement dans les secteurs amont du bassin à risque contribuent à expliquer celles survenant dans la basse plaine occupée par l'agglomération de Sfax. En fait, l'aspect exceptionnel ou catastrophe des inondations de 1982 ne sont que l'expression de la rupture d'équilibre d'un système englobant tout le bassin (Daoud A. 2005). 


\section{Des mesures structurelles: Aménagements d'hydraulique urbaine pour la prévention des risques d'inondation}

11 Mis en place à partir de 1984, les principaux ouvrages avaient pour but l'interception des eaux de ruissellement et leur évacuation vers la mer. Leur description permet de comprendre leur fonctionnement depuis leur réalisation, et d'évaluer leur efficacité eu égard à l'évolution de l'occupation des sols et à la croissance de la périurbanisation anarchique (fig. 2).

Fig. 2 : Dispositif de protection de l'agglomération de Sfax contre les inondations

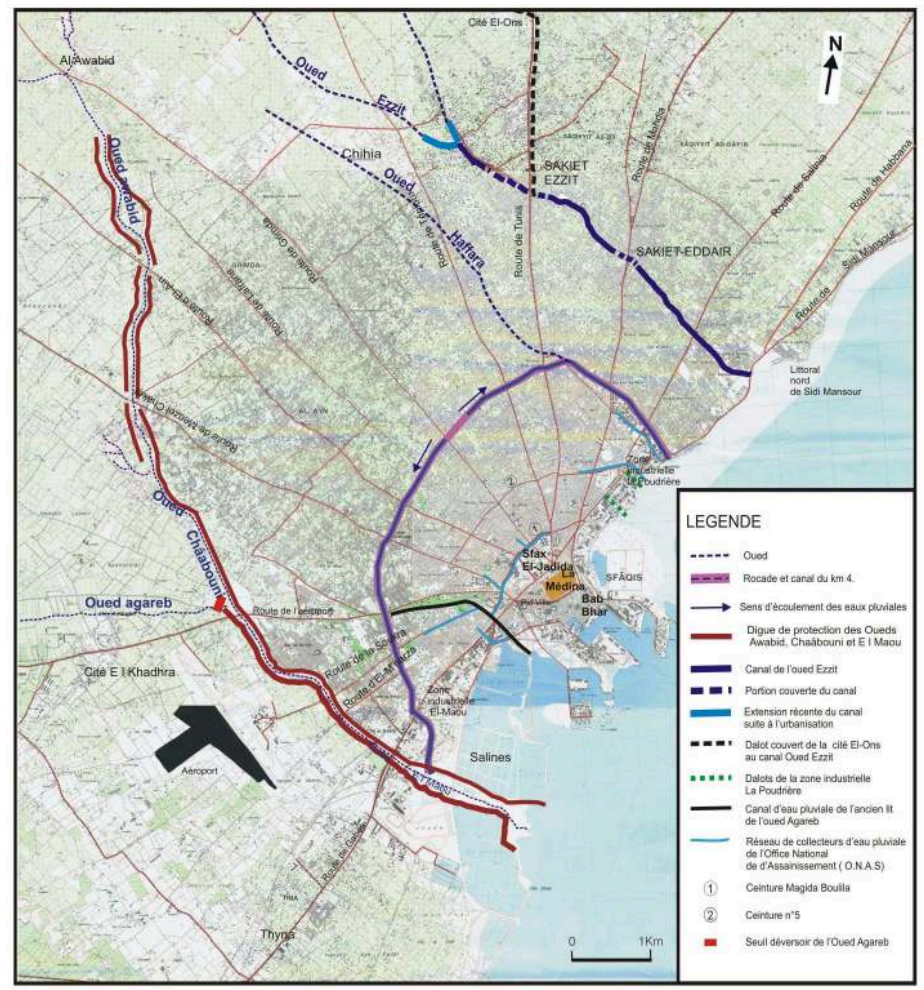




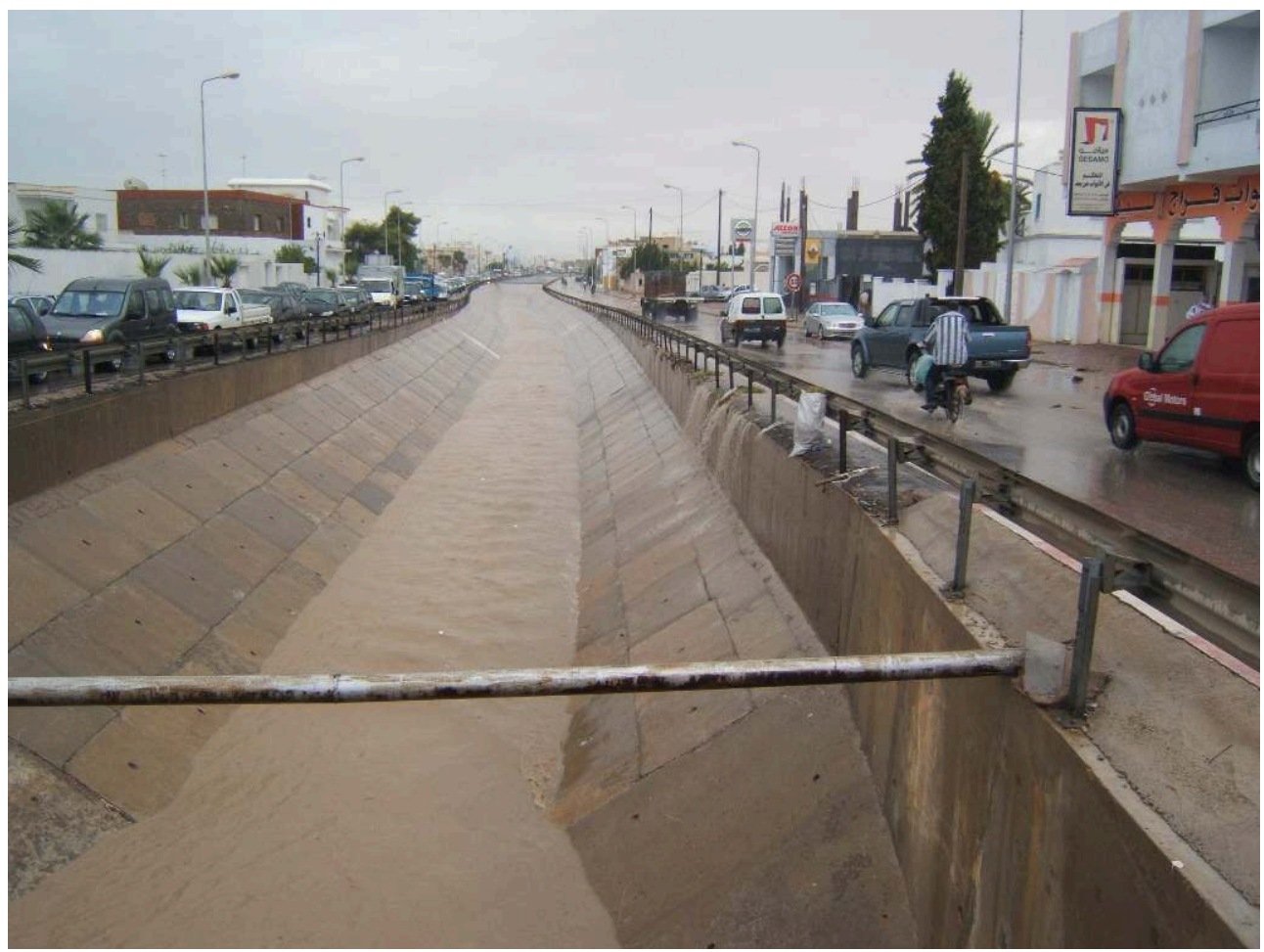

Photo 1 : Canal de la rocade du Km 4

-Le canal de Oued Ezzit : Malgré sa taille limitée, le lit naturel de Oued Ezzit traverse d'abord la commune de Sakiet Ezzit (40.000 habitants), puis la commune de Sakiet Eddaïr $(30.000 \mathrm{~h})$ et coule vers l'Est pour rejoindre la mer. L'aménagement de cet oued a consisté en 1986 en son recalibrage et à sa protection par des digues sur environ $7 \mathrm{~km}$ jusqu'à son embouchure sur le littoral de Sidi Mansour au niveau du Km 6 (photo n 2).

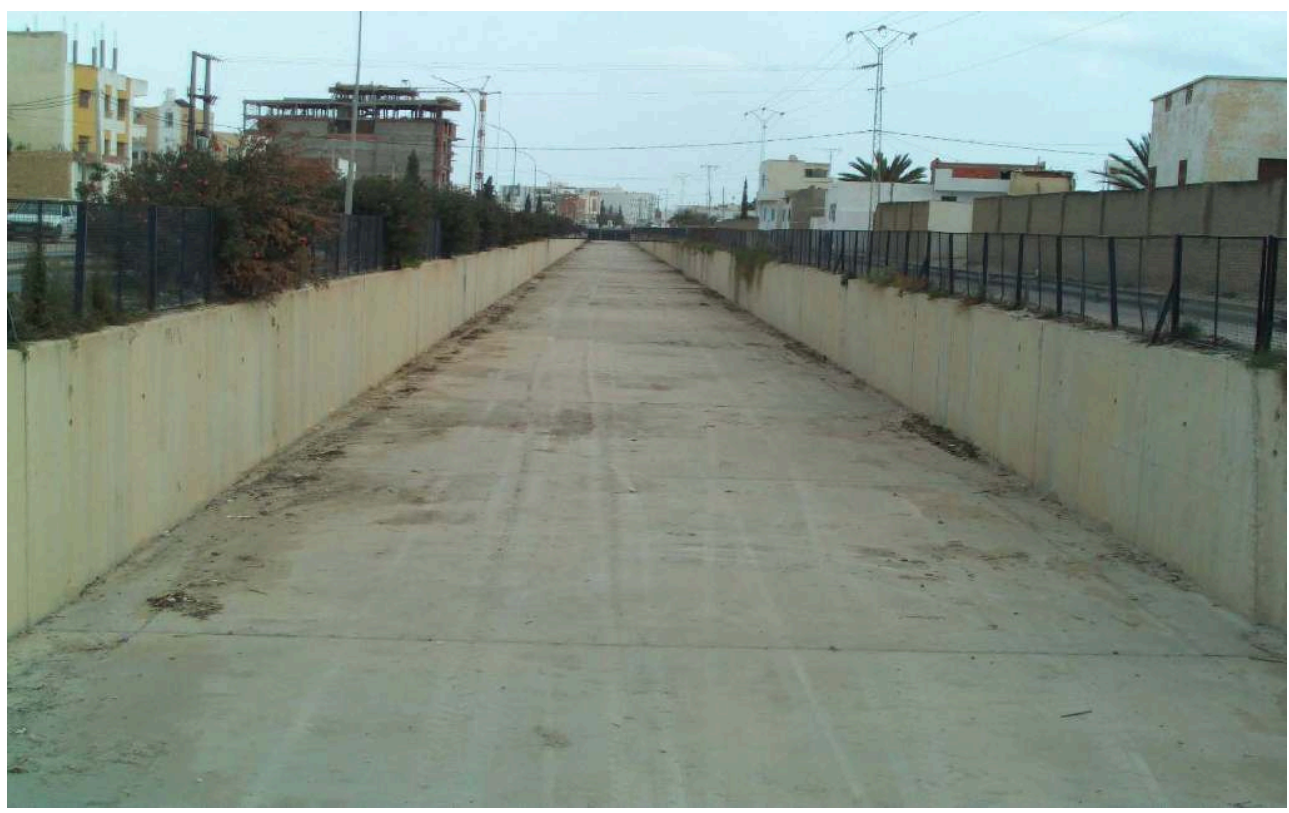

Photo 2 : Canal d'oued Ezzit

13 - La digue de Awabid-Chaabouni-Agareb et El-Maou : Cette digue en terre longe la rive gauche de l'oued El-Awabid-Châabouni et d'El Maou et elle a pour objectif de protéger les zones périurbaines contre les crues. La section aval de la digue, rive gauche 
de Oued El-Maou, commençant au niveau de la route d'Agareb, est ancienne. Elle a été mise en place en 1976, en remplacement d'une digue fusible qui avait sauté lors des inondations de 1969. Elle fut surélevée d'environ 1 mètre en 1985, et doublée d'une deuxième digue sur la rive droite de l'oued. Ce double endiguement est calibré pour recevoir les apports d'eau de Oued Agareb, lui-même branché sur cet ouvrage au moyen d'un ouvrage de dissipation de vitesse de crues. -Dalots des zones industrielles du littoral Nord : Il s'agit d'un système de dalots en réseaux primaire et secondaire, traversant les zones industrielles de la Poudrière I et II, mis en place depuis l'aménagement de la zone au milieu des années 70. -Le réseau de collecte des eaux pluviales en aval du canal de rocade : A l'aval du canal, la collecte et l'évacuation des eaux pluviales se fait essentiellement dans le réseau unitaire et quelques collecteurs d'eau pluviale. Le réseau unitaire comporte essentiellement les collecteurs du centreville, de l'avenue Magida Bboulila et d'une partie des radiales entre celles -ci et la ceinture $n^{\circ} 5$. Il est cependant important de signaler que toute la zone située entre le canal de ceinture et la rocade $n^{\circ} 5$ est dépourvue de réseau de drainage des eaux pluviales.

\section{Différenciation spatiale actuelle des vulnérabilités dans l'agglomération de Sfax}

\section{Transfert vers le péri-urbain de la vulnérabilité physique face au risque d'inondation}

14 L'étalement urbain à Sfax aujourd'hui (Bennasr A. 2004 et 2005) est tel que les infrastructures de protection mises en place au milieu des années 1980 du siècle dernier, bien qu'importantes, coûteuses et efficaces, ne suffisent plus à faire face à l'évolution des vulnérabilités. En effet, depuis une vingtaine d'années, l'agglomération connait un processus de périurbanisation sans précédent. Celui-ci est différent, dans ses formes et son contenu, de celui observé dans les pays développés. Pour le cas de Sfax, les quartiers périurbains abritent des populations d'origine rurale travaillant souvent dans la ville, mais n'arrivant pas à s'y loger, ou des populations urbaines à faibles revenus. Si certains quartiers périurbains sont exposés à des nuisances provenant de la pollution industrielle (Baklouti A. 2004 et 2010), d'autres sont exposés à des risques d'inondation, puisque localisés sur des sites dangereux en bordures d'oueds, comme les quartiers d'El-Awabid, Sidi Salah et Ayoun El-Mayel, ou dans des zones d'expansion des crues comme ceux de Sidi Mansour, El-Khadhra, Aguerba et Erriadh (Fig. 3). Il est évident que ces quartiers sont un indicateur de l'inadaptation des plans d'aménagement urbain à la réalité du terrain, et l'incapacité de la ville à planifier l'accueil des populations de ces quartiers dans des conditions décentes d'accès aux services urbains, ainsi que leur protection contre les risques urbains en général, et d'inondation en particulier. Outre leur vulnérabilité face au risque d'inondation, les populations des quartiers populaires périurbains sont socialement et économiquement vulnérables. La précarité du bâti, du réseau de voierie et la mauvaise accessibilité de ces quartiers sont des facteurs supplémentaires de vulnérabilité, rendant par exemple très difficile l'organisation des secours suite à une catastrophe. Cette vulnérabilité se traduit à travers leur incapacité d'accéder à tes terrains constructibles dans des zones couvertes par les plans d'aménagement, et par le sentiment d'exclusion urbaine qu'ils ressentent. Cette fracture socio-spatiale réduit la résilience de ces populations face au 
risque d'inondation, et accroit leur vulnérabilité face aux dommages matériels qu'ils pourraient subir (submersion rapide de leurs quartiers ou de leurs foyers), ou au dommage moral pouvant résulter de leur sentiment d'être les « laissés pour compte " de l'urbanisation.

\section{Vulnérabilité liée à l'étalement de l'agglomération et à l'organisation actuelle du système de transport urbain}

La concentration des enjeux en zone centrale et péricentrale de plus en plus exigües, peut être considérée aujourd'hui comme le principal facteur de vulnérabilité urbaine. Cela explique l'aggravation des effets des aléas quand ils surviennent. Pour le cas de Sfax, le schéma radioconcentrique des principales voies structurantes et la centralité des activités expliquent certains aspects de sa vulnérabilité. Un bref aperçu historique permet d'expliquer ce constat. (Daoud A et Dahech S. 2009). En effet, jusqu'au début du XIX ème siècle, la médina avait constitué l'élément principal de l'espace, concentrant la quasi-totalité des fonctions d'habitat et d'activités qui généraient peu de flux et nécessitaient peu de déplacements. Elle était entourée par une zone de vergers jardins (jneins) avec des habitats très dispersés, loin des zones inondables. Pendant la colonisation, et vers les années 1930, plusieurs éléments nouveaux(en plus de la construction de deux rocades) vont apparaître dans le paysage urbain : construction du port, configuration du réseau routier en radiales semi-radioconcentriques, naissance de la ville européenne entre la médina et le port, sur des espaces gagnés aux dépens de la mer. A la fin de la colonisation, vers 1955, l'espace urbain de l'agglomération était marqué par la multiplication et la densification des faubourgs, l'extension de la ville européenne et du peuplement européen. De plus, le réseau de radiales devint plus élaboré, et aussi apparition de deux rocades ( $\mathrm{Km} 4$ et $\mathrm{n}^{\circ}$ 5), et émergence des Merkez, littéralement : centres, noyaux d'habitat plus dense sur les radiales, à 4-5 ou 6 kilomètres du centre-ville, parallèlement à la densification des jneins. (Megdich, 1985). Les années $70 \mathrm{du}$ siècle dernier marquent un véritable tournant : densification plus poussée des Jneins, qui subirent des morcellements continus, et qui devinrent des lieux de résidence permanents (Karray, 1983). Les flux pendulaires quotidiens entre la zone centrale et la périphérie résidentielle allaient s'intensifier progressivement, au fur et à mesure du renforcement de la centralité et de la densification de l'habitat en périphérie et de l'étalement urbain. L'opération d'aménagement urbain la plus spectaculaire fut sans doute celle de Sfax El jadida, (nouvelle Sfax), sur les terrains anciennement occupés par les cimetières, jouxtant la médina (sur environ 66 hectares). La ville devint ainsi poly-centrique avec la médina, la ville européenne et Sfax El Jadida (Kallel, 1993 ; Bennasr 2003, 2004). Pour le cas de l'agglomération Sfax, le déséquilibre est énorme entre l'évolution rapide des modes de vie et les exigences de mobilité quotidienne entre zones d'activités et zones de résidence d'une part, et la capacité des réseaux, surtout celui des transports, à suivre le même rythme. Le sous-équipement en infrastructures, la déficience des transports collectifs et l'engorgement rapide des voies principales de circulation sont des traits caractéristiques de l'agglomération. Dans son analyse des relations entre urbanisation et risques, Pigeon P. (2005) affirme qu'outre « les critères classiques concernant les densités de peuplement » qui aggravent la vulnérabilité, « la mobilité accrue des personnes et des biens en milieu urbain l'accentuent ». L'étalement urbain et la diversité des activités de production ou de commandement de l'agglomération de Sfax ont profondément modifié les enjeux et les vulnérabilités, 
particulièrement à travers l'apparition de nouveaux enjeux qualitatifs, liés aux interconnexions des différents réseaux assurant le fonctionnement de la ville. Les inondations de septembre 2009, survenant à une heure de pointe caractérisée par des flux importants de véhicules quittant le centre-ville vers sa périphérie avaient causé en quelques minutes l'interruption des flux. En effet, les trombes d'eau (105 mm en 35 minutes) qui s'étaient abattues sur la ville entrainèrent vite l'engorgement du réseau d'évacuation des eaux pluviales. Le ruissellement urbain et la stagnation de lames d'eau approchant parfois un mètre dans certains nœuds de circulation, avaient fait le reste. La déficience des transports publics et leur inadaptation aux besoins réels de mobilité de la population urbaine expliquent le recours de la population à d'autres moyens, particulièrement les deux roues et la voiture individuelle, que l'infrastructure routière urbaine arrive déjà très mal à contenir, en dehors des situations de crises, lors des heures de pointe.

Fig.3 : Localisation des quartiers populaires périurbains soumis aux risques d'inondation

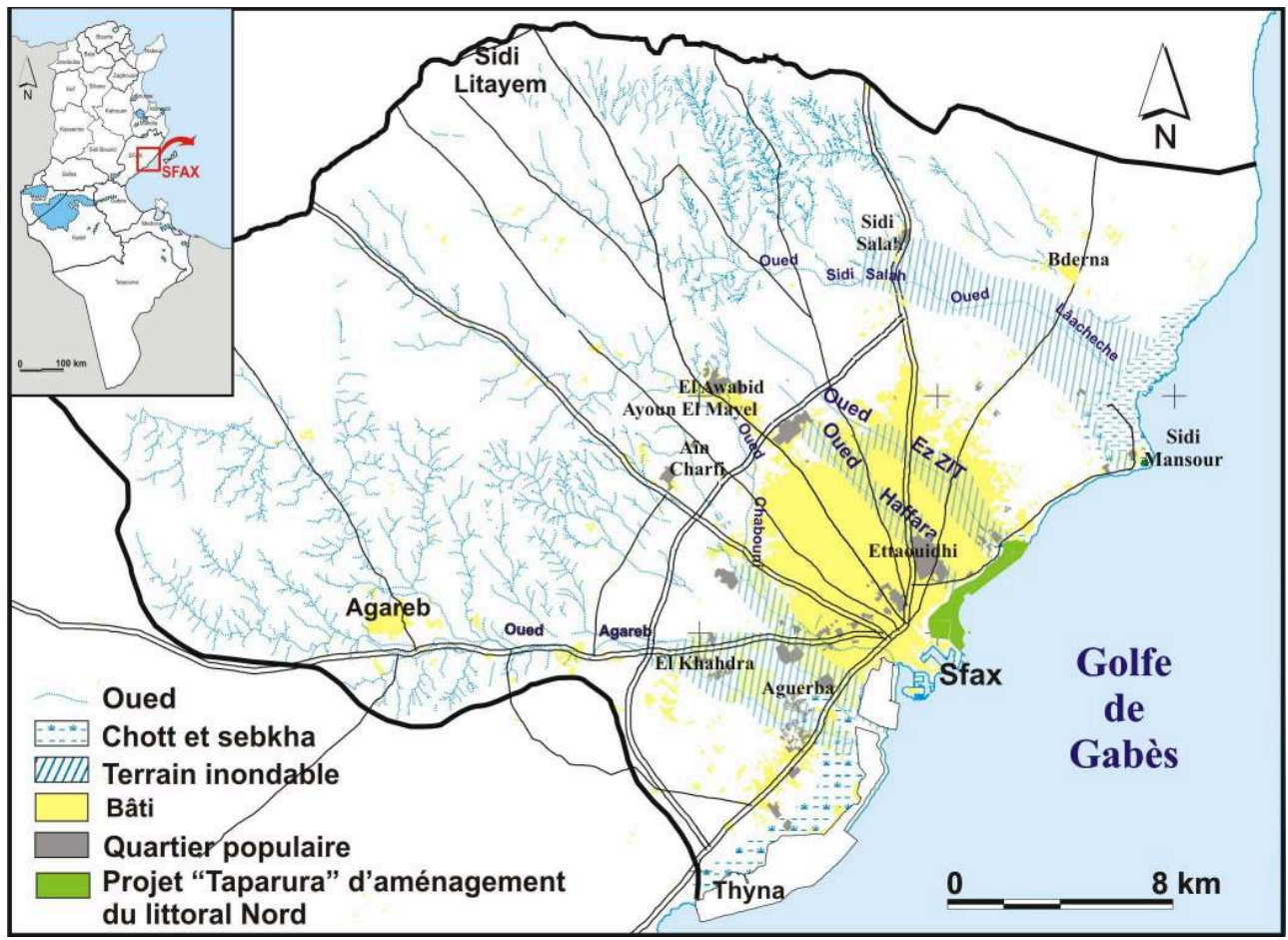

\section{Pour une meilleure articulation entre risque d'inondation et territoire}

\section{L'inscription spatiale des risques d'inondation dans l'agglomération de Sfax}

L'évolution spatio-temporelle des vulnérabilités invite à une réflexion sur une meilleure articulation entre risque d'inondation et territoire. Laganier R. (2006) entend la territorialisation du risque dans le sens "d'une meilleure prise en compte de ce risque dans les documents d'urbanisme et d'aménagement du territoire » De son coté, November V. (2002) affirme « qu'il ne devrait plus être possible d'étudier le risque sans le territoire, ni le territoire sans le risque...cela signifie que les études géographiques du risque doivent tenir compte de la relation riche et complexe entre ces deux éléments. Si 
elles s'y refusent, elles devraient alors prévenir qu'elles ne prennent en compte qu'une vision partielle du territoire, à savoir son rôle de support du risque ". En effet, le risque d'inondation tel qu'il se présente aujourd'hui dans l'agglomération de Sfax, devrait être analysé et géré en relation avec les interdépendances entre zones centrales et périphériques, réseaux de transport et flux d'une part, et ruissellements dans le bassin de risque en général et en milieu urbain en particulier. Les très forts enjeux exposés aux risques d'inondations touchant la zone centrale et péricentrale de l'agglomération de Sfax justifient la nécessité d'une gestion territoriale de ce risque. Les inondations de 2009, ayant en effet entrainé une stagnation d'eau subite et prolongée dans la zone centrale et péricentrale de l'agglomération de Sfax, ont montré, outre la vulnérabilité de ces zones, la fragilité de tout le système territorial duquel dépend l'agglomération, matérialisé par les différents types de réseaux et des liens économiques et humains qu'entretient ce système avec la partie centrale de l'agglomération. L'Etat qui fut jusque-là très centralisateur en Tunisie, a toujours laissé entendre, par la centralisation qu'il exerçait, qu'il était le seul responsable de la sécurité des personnes et de leurs biens en cas d'inondation, mais les évènements récents ont montré que l'ampleur des inondations en milieu urbain dépasse désormais sa capacité de réaction. La paralysie quasi-totale du centre-ville et la submersion de nombreux quartiers populaires situés sur le littoral nord de l'agglomération (particulièrement cité Ettaouidhi), suite aux inondations de septembre 2009 révélèrent que le seuil d'acceptation de ce risque par la population fut dépassé eu égard aux pertes matérielles générées par l'évènement pluvieux. Ainsi, il nous semble utile aujourd'hui de dépasser l'approche par les aléas (qui a conduit à la mise en place des aménagements de protection), et agir désormais sur les vulnérabilités de manière concertée, impliquant, à travers une démarche participative, tous les acteurs du territoire. Cette démarche nécessite une pratique réelle de démocratie locale, que la nouvelle donne du lendemain de la révolution tunisienne du 14 janvier 2011 semble pouvoir concrétiser.

\section{Quels outils pour la gestion future des vulnérabilités face aux risques d'inondation?}

17 Les crues de 1982 (tout comme celles de 1969, non évoquées dans le présent travail), sont restées dans la mémoire collective en raison de leur aspect " catastrophe » car furent accompagnées de pertes en vies humaines. Ces évènements ont conduit les autorités de l'Etat à mettre en place un ensemble de mesures structurelles de gestion des risques dans l'agglomération de Sfax (canal, digues, dalots en milieu urbain, banquettes et autres moyens de contrôle des ruissellements dans les parties amont des bassins versants etc..) destinés à agir sur les aléas. Il nous semble que ces mesures sont arrivées à leurs limites, et que toute extension ou multiplication d'ouvrages risque de devenir économiquement peu supportable pour la communauté. En tout état de cause, ces infrastructures de protection seraient vite dépassées au fur et à mesure que l'étalement urbain anarchique touche de nouvelles zones à risque, non protégées. Fautil dans ce cas étendre de façon continue la protection malgré son coût élevé, ou bien faudrait-il inverser la démarche et contrôler l'urbanisation et l'occupation de l'espace en fonction des risques ? La seconde démarche nous semble plus rationnelle. Elle consisterait à privilégier les mesures non structurelles, c'est-à-dire celles ayant pour objectif d'agir sur les vulnérabilités. Concernant la vulnérabilité physique des secteurs périurbains face aux risque d'inondation, ces mesures devraient infléchir la tendance 
actuelle de prolifération de l'urbanisation anarchique, de réhabiliter et " mettre à niveau " ces quartiers, et de concevoir des outils d'aménagement urbain, aussi bien dans leur volet technique que règlementaire, capables de préserver les berges d'oueds ou les zones d'épandage naturel des crues, de toute urbanisation sans protection préalable. Il va sans dire que cela nécessite une parfaite caractérisation de l'aléa dans ces zones, ainsi que de sa cartographie selon des niveaux d'intensité. Quant à la vulnérabilité fonctionnelle (Léone $\mathrm{F}$ et $\mathrm{Al}, 2010$ ) des zones centrales et péricentrales, ces mesures devraient aider à gérer la crise quand elle survient, à travers des outils comme « les plans de gestion de situation de crise » consistant en un ensemble d'interventions visant à mettre en sécurité les personnes et leurs biens, à travers des mesures spécifiques comme des plans de circulation évitant les secteurs inondés susceptibles de piéger les automobilistes, et de rétablir au plus vite le fonctionnement normal des réseaux dans la ville. Pour le cas de Sfax, la maîtrise des flux de circulation automobile au moment d'un évènement pluvieux exceptionnel nous semble être un élément fondamental de la réduction des vulnérabilités en zone centrale et péricentrale. Ceci passe par l'amélioration de la capacité de réponse individuelle et collective face au risque, et le déclenchement rapide d'un plan de secours visant à éviter les sites à congestion rapide au moment de l'évènement pluvieux, préalablement identifiés, pour organiser ensuite les opérations d'évacuation des eaux. A ce titre, Léone et Al.(idem) notent que « la distinction entre trois niveaux de vulnérabilité (et donc de risques)matérielle, structurelle et fonctionnelle-s'avère nécessaire pour appréhender la complexité systémique des risques urbains et mieux mettre en évidence le rôle de facteurs aussi divers que l'organisation du travail des entreprises, le maillage des réseaux de transport, les modes de prise en charge sociale des personnes fragiles... » De leur côté, Thouret J-C. et D'Ercole R (1996), évoquant la vulnérabilité urbaine, préconisent sa réduction "prévisionnelle avant une crise ", ainsi que sa "réduction opérationnelle pendant le sinistre " et enfin sa "réduction prévisionnelle et opérationnelle après la catastrophe ". A ses différents stades, cette approche nous semble transposable à notre cas d'étude. Toutefois, les documents d'urbanisme (Meat. 1995) ou les études stratégiques (Charfi F. 2008) relatifs à l'agglomération de Sfax, n'ont réservé qu'une place très limitée à la gestion des risques et vulnérabilités. Les outils, qui restent à inventer, pourraient s'inspirer des SDAGE, en France, qui préconisent une planification stratégique de la gestion des ressources en eau à l'échelle de la région ou des grands bassins versants, et des SAGE, qui préconisent des actions à l'échelle locale, incluant, en plus des risques d'inondation, la pollution des eaux et l'érosion, ou enfin des PPRI. Cet emboîtement d'échelles permettrait de saisir la complexité spatiale des risques d'inondation car les conséquences économiques d'un évènement pluvieux exceptionnel peuvent dépasser l'espace directement touché par l'évènement, en raison de l'interdépendance d'enjeux parfois spatialement éloignés.

\section{Conclusion}

Le recours à une approche rétrospective sur les inondations en milieu urbain dans l'agglomération de Sfax a permis de montrer que les vulnérabilités des trois dernières décennies sont différentes de celles d'aujourd'hui. Si l'évènement de 1982 révéla la fragilité physique liée au site inondable, celui de 2009 a permis de mettre en lumière la fragilité du système urbain de l'agglomération de Sfax et révélé au grand jour des vulnérabilités jusque-là sous estimées en zone centrale et péricentrale de la ville, 
théoriquement protégées par le canal de ceinture. La complexité actuelle des vulnérabilités urbaines face au risque d'inondation justifie d'aller au-delà de l'approche par l'aléa, et considérer ce risque comme un système dont la gestion devrait impliquer tous les acteurs de l'agglomération, même si l'action coercitive de l'autorité publique devrait s'exercer encore. L'action de l'Etat dans la gestion du risque d'inondation dans le bassin de Sfax fut guidée depuis trois décennies par la volonté de maîtrise des ruissellements à travers des actions de génie civil et d'hydraulique urbaine. Mais face à l'évolution des vulnérabilités en milieu urbain, il est temps maintenant d'infléchir la tendance en privilégiant les mesures visant à réduire les vulnérabilités, à travers une approche participative. Celle-ci devrait impliquer tous les acteurs du territoire et viser à mettre en place les outils règlementaires de gestion du risque d'inondation et les modalités de fonctionnement des réseaux en milieu urbain en cas de crise. Elle devrait également activer l'intercommunalité à l'échelle de l'agglomération pour la mise en place d'une politique de gestion des risques et de réduction des vulnérabilités.

\section{BIBLIOGRAPHIE}

Baklouti A., (2004) - Les quartiers périurbains du Grand-Sfax : formation, fonctionnement et aménagement. Thèse de Doctorat, Université de Tunis I, Faculté des Sciences Humaines et Sociales

Bennasr, A. (2004) -L'étalement urbain de Sfax. In Revue tunisienne de géographie, 36, 39p. Bennasr, A. (2005). Aménagement urbain durable et gouvernance : Le cas de Sfax. In Colloque SYFACTE/ GREGUM « Les villes au défi du développement durable », Sfax, 2005. < http://www.univlemans.fr/ lettres/labo/gregum/activites/colloques/sfax/axe1/bennasr_ali.htm>,15p.

Charfi, F. (2008). Stratégie de Développement du Grand Sfax, phase IV. Rapport de synthèse. Texte non publié. Municipalité de Sfax.

Bouaziz R. (2011)-Les oueds des environs de Sfax (Tunisie orientale). Editions Universitaires Européennes. 219p.

Bourgou M. et Oueslati A. (1984) -Les conséquences morphologiques des inondations d'octobre 1982 dans le Grand Sfax. In Revue Tunisienne de Géographie. n¹2 ; pp. 157 -170.

Daoud A. (1992) -A propos des inondations exceptionnelles d'octobre 1982 à Sfax. In Revue Tunisienne de Géographie. ; 21/22 ; p. 11-31.

Daoud A. (2005) - Périurbanisation et risque d'inondation. Cas de l'agglomération de Sfax (Tunisie méridionale). In Espaces Maghrébins, Revue de l'Union Géographique Marocaine. n 5-6. pp. 97-112.

Dlala H. (1995) -L'aménagement du Grand Sfax : enjeux, jeu des acteurs et projet de ville. In Cahiers de la méditerranée. Pub. Univ. Nice Sophia Antipolis. n 51. pp 113 -143.

Dlala H. (1996) -Le Grand Sfax : dynamique morpho-fonctionnelle récente et aménagements. In Annales de Géographie. Pub. A. Colin. n 590. pp 369-394.

Karray, N.(1982) Le grand Sfax: Aménagement récent et développement futur. Thèse de Doctorat d'Etat. 2 volumes. Université de Paris-Sorbonne. 
Karray N. (1982) -Le Grand Sfax : Evolution récente, développement futur. Thèse d'Etat. Univ. Paris I. 3 volumes. (ronéo).

Karray N. (1983) -Urbanisation et inondation à Sfax. In L'Ingénieur Tunisien; nº 4; p. 34 -41.

Laganier R (Ed), (2006) Territoires, inondations et figures du risque. L'Harmattan. Itinéraires géographiques. Paris. 257p.

Lamarre D. ; Pagney P. (1997) -Climats et sociétés. Coll. U. Géographie. A. Colin.

Léone F. ; Meschinet De Richemond N. et Vinet F. (2010) Aléas naturels et gestion des risques. Coll. Puf Licence. 287 p.

Megdiche, T. (1985) -Les processus de la croissance urbaine dans la périphérie de Sfax, Thèse de troisième cycle, Université de Toulouse Le Mirail. 272 pages.

Ministère de l'Environnement. France. (1994) -Ruissellement pluvial urbain. Guide de prévention. La Documentation Française.

Ministère de l'Environnement et de l'Aménagement du Territoire. MEAT. Audec Bureau d'études. (1995) -Schéma Directeur d'Aménagement du Grand Sfax. Rapport de première phase. 199p.

November V. (2002)-Les territoires du risque : le risque comme objet de réflexion géographique. Pub. Peter Lang. Bern.332p.

Oueslati A. (1999) -Les inondations en Tunisie. Publication à compte d'auteur. Tunis. B.P. 142. Bardo. 206p.

Pigeon P. (2005) - Géographie critique des risques. Economica. Anthropos. 217 p.

Sakiss N. et Sassi N. (1982) -Les inondations d'octobre 1982 à Sfax: Analyse statistique des données pluviométriques. Institut National de la Météorologie. Tunis. 19p. (ronéo).

Thouret J-C. , D'Ercole R (1996) - Vulnérabilité aux risques naturels en milieu urbain. In Cah. Sci. Hum. $\mathrm{N}^{\circ} 32$. pp. 407-422

\section{RÉSUMÉS}

Située sur le littoral sud-oriental de la Tunisie, l'agglomération de Sfax, implantée dans une plaine littorale vers laquelle convergent plusieurs oueds, compte aujourd'hui plus de 500.000 habitants et s'étale sur environ 22.000 ha. Elle constitue le deuxième pôle économique du pays après la capitale, Tunis. Bien qu'appartenant à la zone semi-aride et ne recevant qu'une moyenne inférieure à $250 \mathrm{~mm}$ de pluies par an, une rétrospective des inondations des 30 dernières années dans l'agglomération de Sfax montre que certains évènements pluviométriques exceptionnels causèrent des dégâts considérables en milieu urbain. Aux aléas climatiques s'ajoute une forte vulnérabilité liée au site, aggravée par des modes d'urbanisation anarchiques ou inappropriés. L'hypothèse principale de recherche dans cette communication est qu'en 30 ans, la vulnérabilité de l'agglomération de sfax face au risque d'inondation reste toujours forte, mais a changé d'aspect, et a évolué dans le temps et dans l'espace. Pour l'étayer, nous retenons pour l'analyse deux évènements pluviométriques exceptionnels. D'abord, les inondations d'octobre 1982, (177 $\mathrm{mm}$ en quelques heures), véritable catastrophe par les dégâts humains et matériels qu'elles causèrent, révélèrent l'extrême vulnérabilité naturelle du site de l'agglomération de Sfax, ce qui a nécessita la mise en place d'une imposante infrastructure de protection (canal ceinturant la ville pour évacuer les eaux de ruissellement, digues de protection, réseau en dalots enterrés d'évacuation d'eaux pluviales, recalibrage d'oueds etc.). Ensuite celles de septembre 2009 (105 mm en 35 minutes sur le centre-ville), qui révélèrent d'autres vulnérabilités: Vulnérabilités liées au 
mode de fonctionnement de la ville, et particulièrement le système de transport, nouvelles vulnérabilités naturelles dans certains secteurs périurbains à cause de l'urbanisation anarchique à proximité des lits d'oueds, dont les acteurs appartiennent à des catégories vulnérables du point de vue économique et social. La communication tente enfin de justifier la nécessité d'une nouvelle approche pour la protection et de renforcement de la résilience face au risque pluvial en milieu urbain, adaptée à cette nouvelle situation. Cette approche devrait privilégier les mesures non structurelles : Plans de protection contre les risques d'inondation, opposables aux tiers dans les décisions d'urbaniser, plans de gestion de situation de crise, impliquant sécurité civiles et autres acteurs de la ville. Au total, une approche qui tient compte du risque dans la gestion du territoire de la ville, car c'est à travers une meilleure territorialisation du risque d'inondation et une meilleure gestion des vulnérabilités que l'agglomération de Sfax pourrait s'adapter aux évènements pluviométriques exceptionnels.

Located on the south-eastern coast of Tunisia, the city of Sfax, on a coastal plain towards which several wadis flow, has now over 500,000 inhabitants. It spreads over about 22,000 hectares. It is the second economic center of the country after the capital, Tunis. Although located in a semiarid zone and receiving an average of less than $250 \mathrm{~mm}$ of rainfall per year, a retrospective of the floods in the past 30 years in the city of Sfax shows that some exceptional rainfall events caused significant damage in urban areas.

The high climatic vulnerability of the site is aggravated by uncontrolled or inappropriate urbanization patterns. The main hypothesis of research in this paper is that in 30 years, the vulnerability of the city of Sfax to the risk of flooding remains high, but has a different aspect, and has evolved over time and in space.

To support this, we have chosen to analyze two exceptional rainfall events. First, the floods of October 1982 (177 $\mathrm{mm}$ in a few hours), a real catastrophe owing to the human and material damages caused, revealed the extreme vulnerability of the natural site of the city of Sfax, which necessitated the establishment of an impressive infrastructure protection (channel surrounding the city for storm water runoff, protection dikes, culverts, underground network of storm drainage, recalibration of wadis etc.). Then those of September 2009 (105 mm in 35 minutes downtown), which revealed additional vulnerabilities: in the operating mode of the city, especially the transportation system, and in some natural areas because of uncontrolled suburban urbanization near the wadi beds, whose actors belong to uncontrolled vulnerable social and economic groups. Finally, the paper attempts to justify the need for a new approach to protect and enhance resilience vis-à-vis storm risk in urban areas, adapted to this new situation. This approach should focus on non-structural measures: plans to protect against flood risk, against third parties in the decision to urbanize, and management plans for crisis situations involving civilian security and other actors in the city. In all, an approach that takes into account the risk in the management of the city areas, for it is through better regionalization of flood risk and a better management of the vulnerabilities that the city of Sfax could adapt to exceptional rainfall events.

\section{INDEX}

Mots-clés : Sfax, vulnérabilités, risque d'inondation, territoire

Keywords : Sfax, vulnerability, flood risk, territory 


\section{AUTEUR}

\section{ABDELKARIM DAOUD}

Professeur des Universités (Eau-Risques et Aménagement). Faculté des Lettres et Sciences

Humaines de Sfax. Département de Géographie. Laboratoire Eau Energie Environnement (Lab 3E).Ecole Nationale d'Ingénieurs de Sfax. B.P.1168. Sfax 3000, Tunisie 